\title{
Sistemas de manejo de plantas daninhas na pré-semeadura da soja ${ }^{1}$
}

\author{
Weeds management systems in soybean pre-planting
}

\author{
Jethro Barros Osipe ${ }^{2}$, Emerson da Silva Teixeira ${ }^{3}$, Gizelly Santos ${ }^{2}$, Robinson Osipe ${ }^{4}$, \\ Camila Ferreira ${ }^{5}$, Petrus Barros Osipe ${ }^{6}$
}

\begin{abstract}
Resumo - O trabalho teve por objetivo avaliar a eficiência e o efeito residual de herbicidas, aplicados na operação de manejo, nas modalidades "Aplique - Plante" e "Manejo Antecipado", na cultura da soja. O delineamento experimental adotado foi blocos ao acaso, com quatro repetições, no esquema fatorial $8 \times 2$, sendo oito misturas de herbicidas (com flumioxazin, chlorimuron-ethyl e glyphosate) em dois sistemas de manejo. Para o "Manejo Antecipado" foi efetuada a aplicação de glyphosate 20 dias antes da semeadura, com os tratamentos sendo aplicados dois dias após o plantio. Já para a modalidade "Aplique - Plante" os tratamentos foram aplicados um dia antes da semeadura da cultura. Foram avaliados a eficiência de controle e o efeito residual dos herbicidas sobre Euphorbia heterophylla, Amaranthus hybridus e Commelina benghalensis, além da altura de plantas, estande e produtividade da cultura da soja. Os resultados mostraram que o sistema "Manejo Antecipado" proporcionou melhor controle das plantas daninhas que "Aplique-Plante", além de garantir que a cultura da soja se desenvolvesse em melhores condições, garantindo maior produtividade. A presença de flumioxazin e chlorimuronethyl foi suficiente para garantir o fechamento das entrelinhas da cultura, sem uma aplicação em pós-emergência.
\end{abstract}

Palavras-Chave: Semeadura direta; dessecação; flumioxazin; chlorimuron-ethyl.

Abstract - The study aimed to evaluate the efficiency and residual effect of herbicides, applied in management operations, in modalities "Apply-Plant" and "Anticipated Management" in soybean crop. The experimental design was randomized complete blocks with four repetitions in $8 \times 2$ factorial scheme, with eight herbicides associations (with flumioxazin, chlorimuron-ethyl and glyphosate) in two management systems. For "Anticipated Management" was conducted glyphosate application 20 days before sowing, with treatments being applied two days after planting. For "Apply-Plant" system, treatments were applied one day before crop sowing. It was evaluated the control efficiency and residual effect of herbicides over Euphorbia heterophylla, Amaranthus hybridus e Commelina benghalensis, besides plant height, stand and soybean yield. Results showed that "Anticipated Management" proportionate better weeds control than "Apply-

\footnotetext{
${ }^{1}$ Recebido para publicação em 17/03/2011 e aceito em 13/06/2011.

${ }^{2}$ Eng $^{\circ}$ Agr $^{\mathbf{0}}$, Mestrando do Programa de Pós-graduação em Agronomia da Universidade Estadual de Maringá, NAPD/UEM. E-mail: jethrosipe@ @otmail.com; gizelly@ agronoma.eng.br

${ }^{3}$ Eng $^{\circ}$ Agro $^{\circ}$, Mestrando do Programa de Pós-graduação em Agronomia da Universidade Estadual de Londrina. Email: emerson_teixeira20@yahoo.com.br

${ }^{4}$ Prof. Adjunto, Universidade Estadual do Norte do Paraná - Campus Luiz Meneghel. E-mail: robosipe@ ffalm.br

${ }^{5}$ Eng $^{\circ}$ Agro $^{\circ}$, Mestrando do Programa de Pós-graduação em Agronomia da Universidade Estadual de Ponta Grossa.

E-mail: cferreira87@hotmail.com

${ }^{6}$ Discente do curso de Agronomia, Universidade Estadual do Norte do Paraná - Campus Luiz Meneghel. E-mail: petrusosipe@hotmail.com
} 
Plant", besides ensuring that soybean crop could be able to develop under better conditions, thus guaranteeing greater yield. Flumioxazin and chlorimuron presence was enough to ensure the closing canopy of crop without a post-emergency application.

Key-words: No-tillage; dessecation; flumioxazin; chlorimuron-ethyl.

\section{Introdução}

$\mathrm{Na}$ cultura da soja se destaca a necessidade de efetuar o controle de plantas daninhas, uma vez que estas causam perdas significativas na produtividade da cultura, conforme a espécie, a densidade e a distribuição na lavoura. Para Homechin (1983), a competição ocorre principalmente por água, luz e nutrientes, podendo ocorrer prejuízos indiretos como dificuldade na operação de colheita, prejudicando a qualidade final do produto. Segundo Pittelkow (2009), dependendo do período de convivência, as infestações de plantas daninhas podem afetar os componentes da produção como o número de vagens por plantas e a massa dos grãos, além da produtividade.

$\mathrm{O}$ sistema de plantio direto pode reduzir a densidade de plantas daninhas na área, pois a cobertura morta pode promover a liberação de compostos alelopáticos (Erasmo et al., 2004) e reduzir a disponibilidade de radiação solar (Facelli \& Pickett, 1991). Mesmo assim, ainda são necessárias medidas de controle das plantas daninhas, principalmente com o uso de herbicidas (Marochi et al., 1995; Sá Pereira et al., 1995).

A eliminação das plantas daninhas antes da semeadura da cultura, chamada de manejo ou dessecação, é dependente da ação eficiente dos herbicidas. A aceitação do sistema pelos agricultores está, em grande parte, na dependência do desenvolvimento de herbicidas eficientes, econômicos, e que não afetem a produção e o meio ambiente.

As plantas daninhas que apresentam maior tolerância ao controle químico, especialmente aos dessecantes, ou para àquelas que existem poucos herbicidas de controle efetivo, são problemáticas no sistema de semeadura direta, considerando que este sistema é extremamente dependente do controle químico. Inserem-se nesse contexto plantas como: grama-seda (Cynodon dactylon), capim-amargoso (Digitaria insularis), buva (Conyza spp.), trapoeraba (Commelina spp.), entre outras (Fidelis et al., 2003).

Como estratégias para o manejo de plantas daninhas citam-se os sistemas ApliquePlante e o Manejo antecipado. No primeiro, ocorre a aplicação de um ou mais herbicidas (normalmente de ação sistêmica) imediatamente antes da semeadura da cultura. É um sistema muito utilizado, pois, os agricultores ganham tempo e maximizam a utilização do maquinário da propriedade (Oliveira Jr. et al., 2006). Já o segundo, consiste na aplicação antecipada (15 a 20 dias em relação à semeadura) de um herbicida sistêmico não-seletivo. Posteriormente é efetuada uma segunda aplicação, na véspera ou logo após a semeadura, normalmente com um produto de ação de contato, cuja função é possibilitar a semeadura no limpo e, também, controlar as possíveis rebrotas de plantas que não foram totalmente controladas na primeira aplicação (Constantin et al., 2007).

Constantin et al. (2000) avaliaram a interação entre diferentes sistemas de manejo químico e formas de controle de plantas daninhas após a emergência da soja, e concluíram que o sistema de manejo antecipado possibilitou melhor controle das espécies daninhas e a redução no uso de herbicidas de controle em pós-emergência. Já em áreas com pouca cobertura vegetal, os efeitos dos sistemas tendem a se igualar com 
relação ao desenvolvimento e produtividade da cultura (Constantin et al., 2007).

Assim, o trabalho teve como objetivo avaliar a eficiência e efeito residual de herbicidas aplicados na operação de manejo, nas modalidades Aplique-Plante e Manejo Antecipado, e seus efeitos no desenvolvimento da cultura da soja.

\section{Material e métodos}

No ano agrícola 2008/2009 foi desenvolvido o presente ensaio com a cultura da soja no município de Bandeirantes, PR, em

Tabela 1. Associações de herbicidas e doses dos tratamentos utilizados no experimento.

\begin{tabular}{|c|c|c|}
\hline \multirow{3}{*}{ Tratamentos } & \multicolumn{2}{|c|}{ Dose } \\
\hline & i. a. ou e.a. & p.c. \\
\hline & \multicolumn{2}{|c|}{$\mathrm{g} \mathrm{ou} \mathrm{mL} \mathrm{ha}^{-1}$} \\
\hline 1. glyphosate $^{/ 1}+$ flumioxazin $^{/ 2}$ & $960+50$ & $2000+100$ \\
\hline 2. glyphosate ${ }^{11}+$ flumioxazin $^{12}$ & $960+75$ & $2000+150$ \\
\hline 3. glyphosate ${ }^{/ 1}+$ flumioxazin $^{\prime 2}$ & $960+100$ & $2000+200$ \\
\hline 4. glyphosate ${ }^{/ 1}+$ flumioxazin $^{/ 2}+$ chlorimuron-ethyl $^{13}$ & $960+50+20$ & $2000+100+80$ \\
\hline 5. glyphosate ${ }^{/ 1}+$ flumioxazin $^{12}+$ chlorimuron-ethyl $^{13}$ & $960+75+20$ & $2000+150+80$ \\
\hline 6. glyphosate ${ }^{/ 1}+$ flumioxazin $^{/ 2}+$ chlorimuron-ethyl $^{13}$ & $960+100+20$ & $2000+200+80$ \\
\hline 7. glyphosate ${ }^{/ 1}+$ chlorimuron-ethyl $^{1 / 3}$ & $960+20$ & $2000+80$ \\
\hline 8. glyphosate ${ }^{/ 1}$ & 960 & 2000 \\
\hline
\end{tabular}

$-^{11}=$ Roundup Transorb ${ }^{\circledR} ;{ }^{12}=$ Flumyzin $500{ }^{\circledR} ;{ }^{13}=$ Classic $^{\circledR}$.

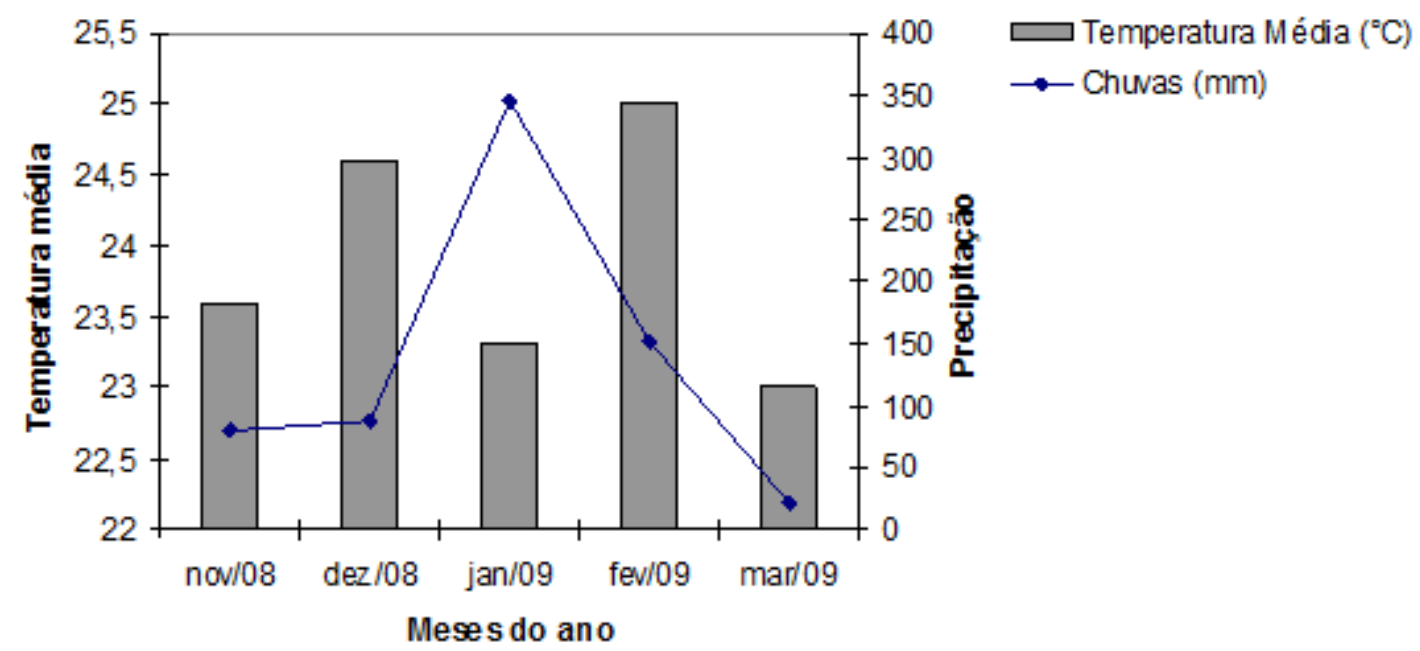


Figura 1. Precipitação $(\mathrm{mm})$ e temperatura média $\left({ }^{\circ} \mathrm{C}\right)$ no período entre novembro de 2008 e março de 2009. Dados obtidos na estação agrometereológica de Bandeirantes, PR. Convênio IAPAR e UENP/CLM.

Os dois sistemas de manejo utilizados foram Manejo Antecipado e Aplique-Plante. As parcelas com tratamentos no sistema Manejo Antecipado receberam aplicação de glyphosate a 960 g e.a. ha $^{-1}$ no dia 22/11/08, e os tratamentos foram aplicados dois dias após a semeadura (DAS), no dia 14/12/08, com temperatura do ar de $28^{\circ} \mathrm{C}$, umidade relativa do ar de $52 \%$, velocidade dos ventos de $2,5 \mathrm{~km}$ $\mathrm{h}^{-1}$ e solo seco. A aplicação dos tratamentos no sistema Aplique-Plante foi realizada no dia $11 / 12 / 08$, com temperatura do ar de $27^{\circ} \mathrm{C}$, umidade relativa do ar $58 \%$, velocidade dos ventos de $2,8 \mathrm{~km} \mathrm{~h}^{-1}$ e solo seco.

A semeadura da cultura da soja foi efetuada no dia 12/12/08, no espaçamento de $0,45 \mathrm{~m}$, utilizando-se 15 sementes por metro linear da cultivar BRS 184, e profundidade média de $0,05 \mathrm{~m}$.

Para a aplicação foi empregado um pulverizador costal pressurizado a $\mathrm{CO}_{2}$, equipado com barra de quatro pontas DG 110.015, montados em corpos com válvula de retenção com diafragma, estando os bicos espaçados um dos outros em $0,50 \mathrm{~m}$. A pressão de trabalho empregada foi de $39 \mathrm{lb} \mathrm{pol}^{-2}$, resultando volume de aplicação de $200 \mathrm{~L} \mathrm{ha}^{-1}$.

As plantas daninhas avaliadas na área experimental e as respectivas porcentagens de cobertura na ocasião da aplicação de manejo eram: Brachiaria plantaginea Link (Hitch) (capim-marmelada) - 30\%; Amaranthus hybridus L. (caruru) - $20 \%$ e Commelina benghalensis (L.) (trapoeraba) - 20\%. Destacase que as plantas daninhas encontravam-se em pleno desenvolvimento vegetativo, entre 10 a $40 \mathrm{~cm}$ de altura, e em alta cobertura do solo.

As avaliações de eficiência dos tratamentos aplicados no manejo foram efetuadas aos 7, 15 e 30 DAS (dias após a semeadura), visualmente mediante comparação com a testemunha sem capina, estabelecendose porcentagens de controle. Avaliou-se também a densidade das plantas daninhas nos diferentes tratamentos mediante a contagem das espécies em quatro quadros de $0,5 \times 0,5 \mathrm{~m}$ por parcela, aos 30 DAE (dias após a emergência), e, ainda aos 60 DAE, foram estabelecidos conceitos de eficiência (Tabela 2), segundo proposta da SBCPD (1995).

A altura do solo até a inserção do último trifólio completamente expandido (10 plantas por parcela) e o estande médio do número de plantas (três amostragens de $3 \mathrm{~m}$ das linhas centrais da área útil) de soja foram avaliados aos 30 DAE. A colheita foi realizada manualmente, tendo sido cortadas as três linhas centrais, desprezando-se $1,0 \mathrm{~m}$ nas extremidades, com posterior trilhagem dos grãos para obtenção da produtividade $\left(\mathrm{kg} \mathrm{ha}^{-1}\right)$.

Os dados obtidos nas avaliações de eficiência de controle, bem como os dados de produtividade, foram submetidos à análise de variância pelo teste $\mathrm{F}$ e para verificar diferença entre médias utilizou-se o teste de agrupamento Scott-Knott, ao nível de 5\% de probabilidade.

\section{Resultados e discussão}

Nas Tabelas 3 e 4 estão os resultados referentes às avaliações de controle na dessecação do caruru e da trapoeraba. $\mathrm{Na}$ avaliação de 7 DAS, o controle das plantas daninhas no sistema "Manejo Antecipado" (M.A.) foi significativamente superior ao sistema "Aplique-Plante" (A.P.) na maioria dos tratamentos. Isso porque, no M.A., a primeira aplicação ocorreu cerca de 20 dias antes da semeadura, havendo tempo suficiente para 
atuação do herbicida. No segundo, a aplicação ocorreu 1 dia antes da semeadura, o que não permitiu que os herbicidas demonstrassem seu potencial de controle aos 7 DAS.

Para Commelina benghalensis, ficou ainda mais evidente diferenças no controle entre os sistemas de manejos. Na avaliação aos 30 DAS, o tratamento 3 (flumioxazin a $100 \mathrm{~g}$ i.a. ha $^{-1}$ ) no M.A. apresentou $98,3 \%$ de eficiência, enquanto que para o mesmo tratamento no A.P., o controle foi de $81,3 \%$.
Essas diferenças são observadas em quase todos os tratamentos (Tabela 4).

Constantin et al. (2009) relatam que a vantagem de uma segunda aplicação, como no Manejo Antecipado, é o fato de que espécies de difícil controle, como a Commelina benghalensis, pode ser adequadamente controlada. Ainda, a segunda aplicação pode servir fundamentalmente para a correção de problemas de rebrotas e de novos fluxos de plantas daninhas já emergidas por ocasião da semeadura (Marochi, 1996).

Tabela 3 - Médias das porcentagens de controle de Amaranthus hybridus aos 07, 15 e 30 DAS, na aplicação de manejo pré-semeadura da soja.

\begin{tabular}{|c|c|c|c|c|c|c|c|}
\hline \multirow{3}{*}{ Tratamento } & \multirow{3}{*}{$\begin{array}{c}\text { Dose } \\
\text { (i. a ou e.a.) } \\
\text { g ou mL ha }\end{array}$} & \multicolumn{6}{|c|}{ Controle $(\%)$} \\
\hline & & \multicolumn{2}{|c|}{7 DAS } & \multicolumn{2}{|c|}{$15 \mathrm{DAS}$} & \multicolumn{2}{|c|}{$30 \mathrm{DAS}$} \\
\hline & & AP & MA & $\mathrm{AP}$ & MA & $\mathrm{AP}$ & MA \\
\hline 1. glyp+flumioxazin & $960+50$ & $84 \mathrm{Bb}$ & $100 \mathrm{Aa}$ & $91 \mathrm{Bb}$ & $100 \mathrm{Aa}$ & $92 \mathrm{Bb}$ & $100 \mathrm{Aa}$ \\
\hline 2. glyp+flumioxazin & $960+75$ & $90 \mathrm{Ab}$ & $100 \mathrm{Aa}$ & $96 \mathrm{Aa}$ & $100 \mathrm{Aa}$ & $98 \mathrm{Aa}$ & $100 \mathrm{Aa}$ \\
\hline 3. glyp+flumioxazin & $960+100$ & $95 \mathrm{Ab}$ & $100 \mathrm{Aa}$ & $98 \mathrm{Aa}$ & $100 \mathrm{Aa}$ & $98 \mathrm{Aa}$ & $100 \mathrm{Aa}$ \\
\hline 4. glyp+flumioxazin + chlorimuron & $960+50+20$ & $91 \mathrm{Ab}$ & $100 \mathrm{Aa}$ & $95 \mathrm{Ab}$ & $100 \mathrm{Aa}$ & $98 \mathrm{Aa}$ & $100 \mathrm{Aa}$ \\
\hline 5. glyp+flumioxazin + chlorimuron & $960+75+20$ & $96 \mathrm{Aa}$ & $100 \mathrm{Aa}$ & $98 \mathrm{Aa}$ & $100 \mathrm{Aa}$ & $99 \mathrm{Aa}$ & $100 \mathrm{Aa}$ \\
\hline 6. glyp+flumioxazin + chlorimuron & $960+100+20$ & $92 \mathrm{Ab}$ & $100 \mathrm{Aa}$ & $96 \mathrm{Aa}$ & $100 \mathrm{Aa}$ & $98 \mathrm{Aa}$ & $100 \mathrm{Aa}$ \\
\hline 7. glyp+chlorimuron & $960+20$ & $92 \mathrm{Ab}$ & $100 \mathrm{Aa}$ & $95 \mathrm{Ab}$ & $100 \mathrm{Aa}$ & $95 \mathrm{Bb}$ & $100 \mathrm{Aa}$ \\
\hline 8. glyphosate & 960 & $79 \mathrm{Bb}$ & $100 \mathrm{Aa}$ & $86 \mathrm{Bb}$ & $100 \mathrm{Aa}$ & $91 \mathrm{Bb}$ & $100 \mathrm{Aa}$ \\
\hline C.V. $(\%)$ & - & & & & & & \\
\hline
\end{tabular}

- AP = "Aplique-Plante"; MA = "Manejo Antecipado"; - As médias seguidas pela mesma, minúscula na linha e maiúscula na coluna, não diferem entre si, ao nível de 5\% de probabilidade pelo teste de agrupamento Scott-Knott.

Tabela 4 - Médias das porcentagens de controle de Commelina benghalensis aos 07, 15 e 30 DAS, na aplicação de manejo pré-semeadura da soja.

\begin{tabular}{|c|c|c|c|c|c|c|c|}
\hline \multirow{3}{*}{ Tratamento } & \multirow{3}{*}{$\begin{array}{c}\text { Dose } \\
\text { (i. a ou e.a.) } \\
\text { g ou mL ha }\end{array}$} & \multicolumn{6}{|c|}{ Controle $(\%)$} \\
\hline & & \multicolumn{2}{|c|}{$7 \mathrm{DAS}$} & \multicolumn{2}{|c|}{$15 \mathrm{DAS}$} & \multicolumn{2}{|c|}{$30 \mathrm{DAS}$} \\
\hline & & $\mathrm{AP}$ & MA & $\mathrm{AP}$ & MA & $\mathrm{AP}$ & MA \\
\hline 1. glyp+flumioxazin & $960+50$ & $64 \mathrm{Bb}$ & $95 \mathrm{Aa}$ & $76 \mathrm{Bb}$ & $98 \mathrm{Aa}$ & $84 \mathrm{Ab}$ & $94 \mathrm{Aa}$ \\
\hline 2. glyp+flumioxazin & $960+75$ & $71 \mathrm{Ab}$ & $91 \mathrm{Aa}$ & $82 \mathrm{Ab}$ & $93 \mathrm{Aa}$ & $80 \mathrm{Ab}$ & $96 \mathrm{Aa}$ \\
\hline 3. glyp+flumioxazin & $960+100$ & $81 \mathrm{Ab}$ & $93 \mathrm{Aa}$ & $80 \mathrm{Ab}$ & $95 \mathrm{Aa}$ & $81 \mathrm{Ab}$ & $98 \mathrm{Aa}$ \\
\hline 4. glyp+flumioxazin + chlorimuron & $960+50+20$ & $74 \mathrm{Ab}$ & $94 \mathrm{Aa}$ & $80 \mathrm{Ab}$ & $98 \mathrm{Aa}$ & $83 \mathrm{Ab}$ & $99 \mathrm{Aa}$ \\
\hline 5. glyp+flumioxazin + chlorimuron & $960+75+20$ & $76 \mathrm{Ab}$ & $96 \mathrm{Aa}$ & $82 \mathrm{Ab}$ & $98 \mathrm{Aa}$ & $86 \mathrm{Ab}$ & $98 \mathrm{Aa}$ \\
\hline 6. glyp+flumioxazin + chlorimuron & $960+100+20$ & $80 \mathrm{Ab}$ & $91 \mathrm{Aa}$ & $82 \mathrm{Ab}$ & $95 \mathrm{Aa}$ & $86 \mathrm{Ab}$ & $94 \mathrm{Aa}$ \\
\hline 7. glyp+chlorimuron & $960+20$ & $68 \mathrm{Ba}$ & $72 \mathrm{Ba}$ & $72 \mathrm{Ba}$ & $78 \mathrm{Ba}$ & $75 \mathrm{Ba}$ & $76 \mathrm{Ba}$ \\
\hline 8. glyphosate & 960 & $58 \mathrm{Bb}$ & $72 \mathrm{Ba}$ & $61 \mathrm{Bb}$ & $72 \mathrm{Ba}$ & $66 \mathrm{Cb}$ & $79 \mathrm{Ba}$ \\
\hline C.V. $(\%)$ & - & \multicolumn{2}{|c|}{9,79} & \multicolumn{2}{|c|}{6,08} & \multicolumn{2}{|c|}{5,60} \\
\hline
\end{tabular}

- AP = "Aplique-Plante"; MA = "Manejo Antecipado"; - As médias seguidas pela mesma, minúscula na linha e maiúscula na coluna, não diferem entre si, ao nível de 5\% de probabilidade pelo teste de agrupamento Scott-Knott. 
Nota-se aos 7 DAS, nas Tabelas 3 e 4, que quando se compara a aplicação do glyphosate isolado e em mistura com flumioxazin + chlorimuron, ocorre maior velocidade de controle das espécies presentes nas misturas testadas, existindo diferenças significativas entre os tratamentos. Esses resultados corroboram com obtidos por Jaremtchuk et al. (2008).

Com relação ao controle de Amaranthus hybridus, observa-se que no sistema M.A. não ocorreram diferenças significativas entre as diferentes misturas de herbicidas (Tabela 3), sendo que todos os tratamentos apresentaram $100 \%$ de eficiência. Ao analisar-se o sistema de A.P., verifica-se que os tratamentos com a mistura tripla de glyphosate + flumioxazin + chlorimuron + superaram significativamente a aplicação de flumioxazin a $50 \mathrm{~g}$ i.a. ha ${ }^{-1} \mathrm{e}$ chlorimuron a $20 \mathrm{~g}$ i.a. ha ${ }^{-1}$. Porém, mesmo com essas diferenças, todos os tratamentos apresentaram controle com níveis aceitáveis. As diferentes misturas de herbicidas no controle de Commelina benghalensis mostraram que, glyphosate isolado e glyphosate + chlorimuron foram significativamente inferiores aos demais tratamentos.

É importante também destacar que a competição e o sombreamento exercido pelas plantas daninhas durante o desenvolvimento inicial pode afetar de forma variada as culturas. Segundo Calegari et al. (1998), esse sombreamento pode afetar negativamente a germinação, a emergência ou o desenvolvimento inicial da cultura, gerando, entre outros efeitos, o estiolamento. Constantin et al. (2007) ressaltam que, em áreas de alta infestação, quanto maior a cobertura do solo pela plantas daninhas no momento da semeadura, maior será o prejuízo à cultura. Já em áreas de baixa infestação, a semeadura poderá ser feita logo após a operação de dessecação, sem prejuízo de produtividade.

Os dados de estande e altura, avaliados aos 30 DAE encontram-se na Tabela 5. Para o estande não existiram diferenças significativas entre os sistemas de manejo, e entre os diferentes herbicidas utilizados. Para a altura, comparando-se os sistemas de manejo, verifica-se que existiram diferenças entre eles em alguns tratamentos. No tratamento 2 (glyphosate + flumioxazin), as plantas de soja do M.A. superaram as do A.P. em 5,4 cm. O mesmo ocorreu também nos tratamentos 4 e 6 (glyphoste + flumioxazin + chlorimron) e 8 (glyphosate).

Segundo Procópio et al. (2006), em áreas com grande cobertura vegetal, as culturas semeadas logo após o controle químico podem apresentar redução no desenvolvimento vegetativo. Monquero et al. (2010), em trabalho com diferentes intervalos de dessecação e semeadura, notaram que coberturas não controladas totalmente podem exercer efeito negativo na germinação da soja, diminuindo o estande e altura da cultura.

A presença dos herbicidas com atividade residual nas aplicações garantiu a cultura o fechamento das entrelinhas sem a necessidade da aplicação em pós-emergência. Nas avaliações de densidade efetuadas, onde havia a presença dos herbicidas flumioxazin e/ou chlorimuron, o número de plantas daninhas foi bem inferior. 
Tabela 5 - Médias referentes ao estande $\left(\right.$ plantas $\left.\mathrm{m}^{-1}\right)$ e altura $(\mathrm{cm})$ das plantas de soja.

\begin{tabular}{lccccc}
\hline \multicolumn{1}{c}{ Tratamentos } & \multicolumn{1}{c}{$\begin{array}{c}\text { Dose } \\
\text { (i.a. ou e.a. }\end{array}$} & \multicolumn{2}{c}{ Estande 30 DAE } & \multicolumn{2}{c}{ Altura 30 DAE } \\
\cline { 2 - 5 } & g ou mL ha & AP & MA & AP & MA \\
\hline 1. glyp+flumioxazin & $50+960$ & $13,4 \mathrm{Aa}$ & $12,2 \mathrm{Aa}$ & $34,5 \mathrm{Ba}$ & $38,2 \mathrm{Ba}$ \\
2. glyp+flumioxazin & $75+960$ & $10,2 \mathrm{Aa}$ & $12,1 \mathrm{Aa}$ & $38,0 \mathrm{Ab}$ & $43,4 \mathrm{Aa}$ \\
3. glyp+flumioxazin & $100+960$ & $11,7 \mathrm{Aa}$ & $12,0 \mathrm{Aa}$ & $37,6 \mathrm{Aa}$ & $41,7 \mathrm{Aa}$ \\
4. glyp+flumioxazin + chlorimuron & $50+960+20$ & $12,1 \mathrm{Aa}$ & $13,2 \mathrm{Aa}$ & $37,5 \mathrm{Ab}$ & $43,2 \mathrm{Aa}$ \\
5. glyp+flumioxazin + chlorimuron & $75+960+20$ & $12,3 \mathrm{Aa}$ & $12,4 \mathrm{Aa}$ & $39,7 \mathrm{Aa}$ & $41,5 \mathrm{Aa}$ \\
6. glyp+flumioxazin + chlorimuron & $100+960+20$ & $10,8 \mathrm{Aa}$ & $12,7 \mathrm{Aa}$ & $40,4 \mathrm{Ab}$ & $44,0 \mathrm{Aa}$ \\
7. glyp+chlorimuron & $20+960$ & $10,9 \mathrm{Aa}$ & $12,1 \mathrm{Aa}$ & $37,9 \mathrm{Aa}$ & $41,9 \mathrm{Aa}$ \\
8. glyphosate & 960 & $11,1 \mathrm{Aa}$ & $13,8 \mathrm{Aa}$ & $37,8 \mathrm{Ab}$ & $42,9 \mathrm{Aa}$ \\
\hline C.V. (\%) & - & \multicolumn{2}{c}{12,29} & & 8,70 \\
\hline
\end{tabular}

- AP = "Aplique-Plante"; MA = "Manejo Antecipado"; - As médias seguidas pela mesma, minúscula na linha e maiúscula na coluna, não diferem entre si, ao nível de 5\% de probabilidade pelo teste de agrupamento Scott-Knott.

Para Amaranthus que a inclusão de herbicidas com atividade superior a $85 \%$ (Tabel 6). Para Euphorbia superior a 85\% (Tabela 6). Para Euphorbia flumioxazin, reduz a interferência de plantas heterophylla (Tabela 7), essa redução foi daninhas no início do ciclo da cultura, podendo menor, porém a diferença foi perceptível no inclusive, contribuir no fechamento mais porte da planta daninha, que se apresentou com efetivo e precoce da mesma.

tamanho reduzido na presença desses

herbicidas. Jaremtchuk et al. (2008) relatam

Tabela 6 - Densidade média de Euphorbia hetorophylla e conceitos referentes ao nível de controle da espécie aos 30 dias após emergência da cultura da soja (DAE), nos sistemas "Aplique-Plante" (AP) e "Manejo Antecipado" (MA).

\begin{tabular}{|c|c|c|c|c|c|}
\hline \multirow[t]{2}{*}{ Tratamentos } & \multirow{2}{*}{$\begin{array}{c}\text { Dose } \\
\text { (i.a. ou e.a.) } \\
\text { g ou mL ha }^{-1} \\
\end{array}$} & \multicolumn{2}{|c|}{ Densidade 30 DAE } & \multicolumn{2}{|c|}{ Conceito $60 \mathrm{DAE}$} \\
\hline & & AP & MA & $\mathrm{AP}$ & MA \\
\hline 1. glyp+flumioxazin & $50+960$ & $4,3 \mathrm{Ba}$ & $1,8 \mathrm{Ba}$ & $\mathrm{B}$ & $\mathrm{B}$ \\
\hline 2. glyp+flumioxazin & $75+960$ & $2,5 \mathrm{Ba}$ & $2,0 \mathrm{Ba}$ & B & B \\
\hline 3. glyp+flumioxazin & $100+960$ & $3,0 \mathrm{Ba}$ & $1,8 \mathrm{Ba}$ & B & A \\
\hline 4. glyp+flumioxazin + chlorimuron & $50+960+20$ & $1,8 \mathrm{Ba}$ & $1,0 \mathrm{Ba}$ & $\mathrm{B}$ & A \\
\hline 5. glyp+flumioxazin + chlorimuron & $75+960+20$ & $4,8 \mathrm{Ba}$ & $2,5 \mathrm{Ba}$ & B & A \\
\hline 6. glyp+flumioxazin + chlorimuron & $100+960+20$ & $2,0 \mathrm{Ba}$ & $0,8 \mathrm{Ba}$ & B & A \\
\hline 7. glyp+chlorimuron & $20+960$ & 7,8 Aa & $2,0 \mathrm{Bb}$ & B & A \\
\hline 8. glyphosate & 960 & $11,5 \mathrm{Aa}$ & $12,0 \mathrm{Aa}$ & $\mathrm{E}$ & $\mathrm{E}$ \\
\hline
\end{tabular}

- Conceito A = Controle Excelente, $\mathrm{E}$ = Ausência de controle; - As médias seguidas pela mesma, minúscula na linha e maiúscula na coluna, não diferem entre si, ao nível de 5\% de probabilidade pelo teste de agrupamento Scott-Knott.

Analisando os conceitos adotados aos enquanto o sistema Aplique Plante recebeu o 60 DAE (Tabelas 6 e 7), percebe-se que o conceito B (Controle bom, aceitável para Manejo Antecipado se mostrou melhor que o infestação da área), o sistema Manejo Aplique Plante, principalmente para o leiteiro. Antecipado recebeu A (Controle excelente ou Nos tratamentos de 03 a 07 (Tabela 7), total da espécie em estudo). A segunda 
aplicação no Manejo Antecipado, efetuada com o solo praticamente sem cobertura vegetal, garantiu melhor atividade residual dos herbicidas.

Segundo Cobucci et al. (2004), alguns herbicidas residuais têm comportamentos diferentes quando utilizados em préemergência sobre a cobertura morta, no sistema de plantio direto. Ao serem aplicados estes deveriam chegar até a superfície do solo. Todavia, uma parte deles é retida pela cobertura, não atingindo o solo.

Tabela 7 - Densidade média de Amaranthus hybridus e conceitos referentes ao nível de controle da espécie aos 30 dias após emergência da cultura da soja (DAE), nos sistemas "Aplique-Plante" (AP) e "Manejo Antecipado" (MA).

\begin{tabular}{|c|c|c|c|c|c|}
\hline \multirow[t]{2}{*}{ TRATAMENTOS } & \multirow{2}{*}{$\begin{array}{l}\text { DOSAGENS } \\
\text { (i.a. ou e.a.) } \\
\text { g ou mL ha }^{-1}\end{array}$} & \multicolumn{2}{|c|}{ Densidade 30 DAE } & \multicolumn{2}{|c|}{ Conceito $60 \mathrm{DAE}$} \\
\hline & & $\mathrm{AP}$ & MA & AP & MA \\
\hline 1. glyp+flumioxazin & $50+960$ & $1,5 \mathrm{Ba}$ & $0,8 \mathrm{Ba}$ & $\mathrm{B}$ & $\mathrm{B}$ \\
\hline 2. glyp+flumioxazin & $75+960$ & $1,0 \mathrm{Ba}$ & $0,3 \mathrm{Ba}$ & $\mathrm{B}$ & A \\
\hline 3. glyp+flumioxazin & $100+960$ & $0,5 \mathrm{Ba}$ & $0,3 \mathrm{Ba}$ & A & A \\
\hline 4. glyp+flumioxazin + chlorimuron & $50+960+20$ & $0,3 \mathrm{Ba}$ & $0,0 \mathrm{Ba}$ & A & A \\
\hline 5. glyp+flumioxazin + chlorimuron & $75+960+20$ & $0,0 \mathrm{Ba}$ & $0,0 \mathrm{Ba}$ & A & A \\
\hline 6. glyp+flumioxazin + chlorimuron & $100+960+20$ & $0,0 \mathrm{Ba}$ & $0,0 \mathrm{Ba}$ & A & A \\
\hline 7. glyp+chlorimuron & $20+960$ & $0,8 \mathrm{Ba}$ & $0,0 \mathrm{Ba}$ & $\mathrm{B}$ & A \\
\hline 8. glyphosate & 960 & $10,3 \mathrm{Aa}$ & $8,3 \mathrm{Aa}$ & $\mathrm{E}$ & $\mathrm{E}$ \\
\hline C.V. $(\%)$ & - & & & & \\
\hline
\end{tabular}

- Conceito A = Controle Excelente, $\mathrm{E}$ = Ausência de controle; - As médias seguidas pela mesma, minúscula na linha e maiúscula na coluna, não diferem entre si, ao nível de 5\% de probabilidade pelo teste de agrupamento Scott-Knott.

A produtividade da cultura da soja foi favorecida pelo M.A. Independentemente das formas de controle utilizadas, comparando apenas e média total dos dois sistemas de manejo, verifica-se queda de $276,17 \mathrm{~kg} \mathrm{ha}^{-1}$, onde foi adotado o A.P. No tratamento 02 (glyphosate a $960 \mathrm{~g}$ e.a. ha $^{-1}+$ flumioxazin a 50 g i.a. $\mathrm{ha}^{-1}$ ) esses valores chegam a $615,3 \mathrm{~kg} \mathrm{ha}^{-1}$ (Tabela 8), havendo diferenças estatísticas.

Constantin et al. (2009) verificaram quedas de produtividade na cultura da soja de até 17,5 sacas por hectare no sistema A.P., quando comparado ao sistema de M.A. Oliveira Júnior et al. (2006) também mostraram que a soja produziu cerca de $562 \mathrm{~kg}$ $\mathrm{ha}^{-1}$ a mais, onde foi utilizado o Manejo
Antecipado. Outros trabalhos, ainda, corroboram tais resultados como Constantin et al. (2005) e Oliveira Júnior et al. (2005).

Para Oliveira Júnior et al. (2006), outros benefícios podem ser constatados com a dessecação antecipada, como maior eficiência e rendimento na operação de semeadura, menos infestação nas culturas estabelecidas em sucessão e melhor eficácia dos herbicidas aplicados após a emergência. Para Greco (2003), a dessecação em época adequada, pode levar a uma maior eficiência e rendimento da semeadora, evitando problemas de embuchamento e dificuldades de corte. 
Tabela 8 - Produtividade média da cultura da soja cultivar BRS 184, submetidas aos tratamentos nos sistemas "Aplique-Plante" (AP) e "Manejo Antecipado" (MA).

\begin{tabular}{lccc}
\hline \multirow{2}{*}{ TRATAMENTOS } & $\begin{array}{c}\text { DOSAGENS } \\
\text { (i.a. ou e.a. }\end{array}$ & \multicolumn{2}{c}{ Produtividade $\left(\mathrm{kg} \mathrm{ha}^{-1}\right)$} \\
\cline { 3 - 4 } & g ou mL ha $^{-1}$ & $\mathrm{AP}$ & MA \\
\hline 1. glyp+flumioxazin & $50+960$ & $2057,22 \mathrm{Bb}$ & $2490,95 \mathrm{Aa}$ \\
2. glyp+flumioxazin & $75+960$ & $2230,20 \mathrm{Bb}$ & $2846,12 \mathrm{Aa}$ \\
3. glyp+flumioxazin & $100+960$ & $2210,70 \mathrm{Bb}$ & $2689,50 \mathrm{Aa}$ \\
4. glyp+flumioxazin + chlorimuron & $50+960+20$ & $2359,90 \mathrm{Aa}$ & $2549,32 \mathrm{Aa}$ \\
5. glyp+flumioxazin + chlorimuron & $75+960+20$ & $2569,50 \mathrm{Aa}$ & $2562,78 \mathrm{Aa}$ \\
6. glyp+flumioxazin + chlorimuron & $100+960+20$ & $2458,10 \mathrm{Aa}$ & $2770,12 \mathrm{Aa}$ \\
7. glyp+chlorimuron & $20+960$ & $2859,97 \mathrm{Aa}$ & $2800,32 \mathrm{Aa}$ \\
8. glyphosate & 960 & $1880,72 \mathrm{Ba}$ & $2126,42 \mathrm{Ba}$ \\
\hline C. V. $\%$. & - & & 10,96
\end{tabular}

- As médias seguidas pela mesma, minúscula na linha e maiúscula na coluna, não diferem entre si, ao nível de 5\% de probabilidade pelo teste de agrupamento Scott-Knott.

\section{Conclusão}

O sistema de Manejo Antecipado se mostrou melhor que o Aplique-Plante, tanto para o controle de Commelina benghalensis, Amaranthus hybridus e Euphorbia heterophylla, quanto para o desenvolvimento da cultura da soja; e a aplicação dos herbicidas flumioxazin, associado ao chlorimuron, foi suficiente para garantir o fechamento das entrelinhas da cultura, sem uma aplicação em pós-emergência.

\section{Referências}

CALEGARI, A. et al. Culturas, Sucessões e Rotações. In: Sistema plantio direto. o produtor pergunta a embrapa responde. Dourados: Embrapa-CPAO. Dourados, 1998, p.59-80.

COBUCCI, T. et al. Economic viability of residual herbicides in dry bean pre-planting under two aplication methods. Planta Daninha, v.22, n.4, p.583-590, 2004.

CONSTANTIN, J. et al. Sistemas de manejo em plantio direto e sua influência sobre herbicidas aplicados em pós-emergência na cultura da soja. Revista Brasileira de Herbicidas, v.1, n.3, p.233-242, 2000.

CONSTANTIN, J. et al. Sistemas de manejo: efeitos sobre o desenvolvimento da soja e sobre o controle de plantas daninhas. In: REUNIÃO DE PESQUISA DA SOJA NA REGIÃO CENTRAL DO BRASIL, 27, 2005, Cornélio Procópio, PR. Resumos... Londrina: Embrapa Soja, 2005, p.527-528.

CONSTANTIN, J. et al. Interação entre sistemas de manejo e de controle de plantas daninhas em pós-emergência afetando o desenvolvimento e a produtividade do milho. Planta Daninha, v.25, n.3, p.513-520, 2007.

CONSTANTIN, J. et al . Sistemas de manejo de plantas daninhas no desenvolvimento e na produtividade da soja. Bragantia, v.68, n.1, p. 125-135, 2009.

ERASMO, E.A.L. et al. Potencial de espécies utilizadas como adubo verde no manejo integrado de plantas daninhas. Planta Daninha, v.22, n.3, p.337-342, 2004. 
FACELLI, J.M.; PICKETT, S.T.A. Plant litter: light interception and effects of an old-field plant community. Ecology, v.72, n.3, p.10241031, 1991.

FIDELIS, R.R. et al. Alguns aspectos do plantio direto para a cultura da soja. Bioscience Journal, v.19 n.1 p.23-31, 2003.

GREGO, C.R.; BENEZ, S.H. Manejo da cobertura vegetal do solo na implantação da cultura da soja (Glycine max (L.) Merril) semeada com dois mecanismos sulcadores. Energia na Agricultura, v.18, n.3, p.48-52, 2003.

HOMECHIN, M. Rotacao de culturas e a incidência de patógenos da soja. Londrina: EMBRAPA-CNPSo, 1983.

JAREMTCHUK, C.C. et al. Efeito de sistemas de manejo sobre a velocidade de dessecação, infestação inicial de plantas daninhas e desenvolvimento e produtividade da soja. Acta Scientiarum Agronomy, v.30, n.4, p.449-455, 2008.

MAROCHI, A.I. Avaliação de métodos de controle químico para Richardia brasiliensis (poaia-branca), infestando áreas sob plantio direto da Região Sul do Brasil. In: ZAPP: Desafio do novo. São Paulo: Zeneca Agrícola, 1996, p.175-186.

MAROCHI, A.I.; MIERLO, C.V.; GALLO, P. Eficiência de flumetsulam aplicado sob diferentes quantidades de palha, em sistema de plantio direto, no controle de dicotiledôneas na cultura da soja. In: CONGRESSO BRASILEIRO DA CIÊNCIA DAS PLANTAS DANINHAS, 20., Florianópolis, 1995. Resumos... Florianópolis: SBCPD, 1995. p. 76-78.
MONQUERO, P.A et al. Intervalo de dessecação de espécies de cobertura do solo antecedendo a semeadura da soja. Planta Daninha, v.28, n.3, p.561-573, 2010.

OLIVEIRA JR., R.S. et al. Efeito de dois sistemas de manejo sobre o desenvolvimento e a produtividade da soja. In: REUNIÃO DE PESQUISA DA SOJA NA REGIÃO CENTRAL DO BRASIL, 27, 2005, Cornélio Procópio, PR. Resumos... Londrina: Embrapa Soja, 2005. p.525-526.

OLIVEIRA JÚNIOR., R.S. et al. Interação entre sistemas de manejo e controle de plantas daninhas em pós-emergência afetando o desenvolvimento e a produtividade da soja. Planta Daninha, v.24, n.4, p.721-732, 2006.

PITTELKOW, F. et al. Interferência de plantas daninhas na cultura da soja transgênica. Global Science and Technology, v.2, n.3, p.543-549, 2009.

PROCÓPIO, S.O. et AL. Efeito de dessecantes no controle de plantas daninhas na cultura da soja. Planta Daninha, v.24, n.1, p.193-197, 2006.

SÁ PEREIRA, E. et al. Avaliações qualitativas e quantidades da comunidade infestante de plantas na cultura de soja (Glycine max), cultivada em plantio direto e convencional e submetida a distintos sistemas de controle das plantas daninhas. In: CONGRESSO BRASILEIRO DA CIÊNCIA DAS PLANTAS DANINHAS, 20., 1995, Florianópolis. Resumos... Florianópolis: SBCPD, 1995. p. 431-432.

SOCIEDADE BRASILEIRA DA CIÊNCIA DAS PLANTAS DANINHAS. Procedimentos para instalação, avaliação e análise e experimentos com herbicidas. Londrina: SBCPD, 1995. 42p. 